\title{
Research on Combinatorial Approach in Composition-structure-property of Non-metallic Materials
}

\author{
Shuhu Li ${ }^{1}$, Danyong Wang ${ }^{1}$, Xiaoyan $\mathrm{Li}^{2}$, Juncong Liu${ }^{1}$, Yiwei Chen ${ }^{1}$, Huamin \\ $\mathrm{Jia}^{1}$, Zhenming Qin ${ }^{1}$, Ronghui Wang ${ }^{1}$, Huazhen Wei ${ }^{1}$ \\ ${ }^{1}$ Shandong Non-metallic Materials Institute, Jinan, China, 250031 \\ ${ }^{2}$ Shandong Urban Construction Vocational College, Jinan, China, 250031
}

Key words: Combinatorial approach; Composition; Structure; Property; Non-metallic materials Abstract: The mechanism of combinatorial approach was reviewed first and then the passive mixers and active mixers for discovering new kinds of non-metallic materials by the use of combinatorial approach were introducted. By active or passive mixing, combinatorial approach was used to find close relatinship butween structrure and property in order to dip out and find new products or new properties of materials. Cases of combinatorial approach were reviewed. Combinatorial approach had been used in multiple ranges, such as discovering new kinds of chemical materials, researching new classes of biological-medical materials and so on. The practical corresponding conclusions were made at last.

MGI, Materials Genome Initiative, which is an initiative declared by USA president Obama has aroused intensive interests of materials specialists all around the world since 2011. With the central contents of Computing tools, Experimental tools and databases, MGI is proposed to serve for national safety, human beings wellfare and so on. However, the models of materials or products could be achieved by computing tools and the most important and core contents of MGI are to find the relationships between micro- and macro- structures and properties of materials by using experimental tools. The combinatorial approach of metal or non-metal materials is the means to achieve the above goal and it was reviewed in this paper so as to provide guidance for materials' discovery. The two important foundaments of MGI including the first principle for computing and high-throughout experiment method put forward the fast development of new-materials' discovery. That means, on one hand, MGI is ready to solve numerous micro- and macro- materials problems from the view of the first principle that means solving problems begins with atom-structure point as the basis of reach into materials. On the other hand, MGI combines high-throughout experiments with first-priciple together to achieve new kinds of materials' discovery.

Many means include high-throught experiment integrated materials combinal-chip approach, diffusion multiple approach and additive manufacturing (3D printing) are used to solve the problems. The high-throughout expeirmental techniques are to be interviewed in this paper so as to make a solid foundation for new non-metallic materials' discovery and provide guidance for materials researchers to develop new kinds of materials.

\section{Mechanism of Combinatorial Approach}

Meredith et al ${ }^{[1]}$ demonstrated the power of combinatorial approaches to mapping polymer phase diagrams, by developing an efficient way to make polymer composition gradient films with three phases including gradient mixing, deposition and film spreading as shown in Fig.1. 


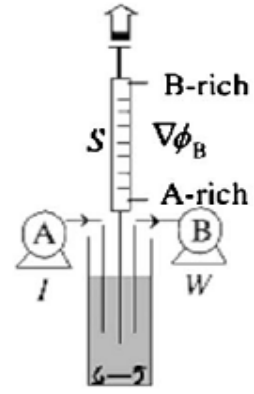

Mix compositiongradient column (a)

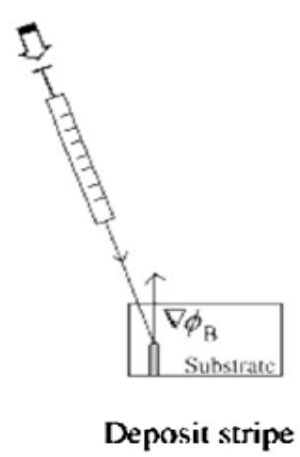

(b)

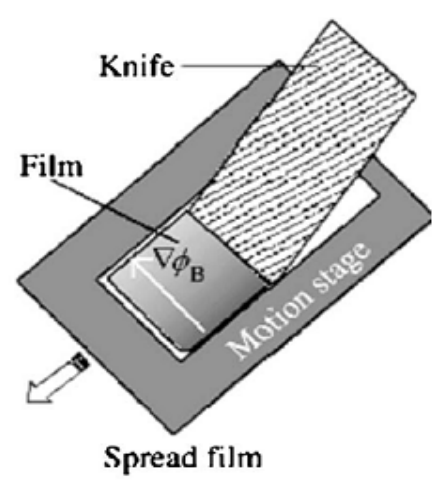

(c)

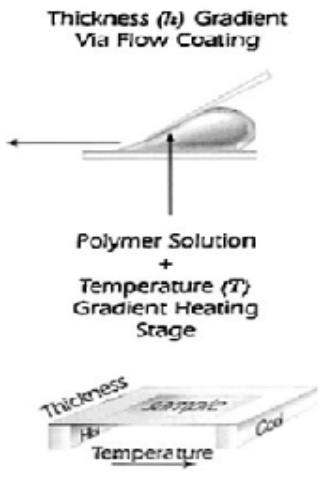

(d)

Fig.1 Polymer-phase diagrams-mapping

Fig. 1 showed various different methods of making polymer combinatorial spreads/libraries: (a)-(d) steps in making continuous polymer film gradients with composition, thickness and temperature variations ${ }^{[1-4]}$.

\section{Research on Combinatorial Approaches}

Mixing viscous liquids in microfluidic systems is a challenging task. During the task, there are two kinds of mixers including passive mixers and active mixers. The former is complex channel geometries or heterogeneous surface to enhance diffusion or generate chaotic advection ${ }^{[5]}$ and the latter is disturbance generated by the external field (pressure, thermal, electrokinetic, magnetic and acoustic) for enhancing mixing ${ }^{[6,7]}$.

Meredith et al mixed polystyrene (PS) and poly(vinyl methyl ether) (PVME) and the loss of transparency in the PS-PVME binary system could be directly seen with the naked eye from the combinatorial polymer film in a temperature gradient, as shown in Fig.2. And the image itself represents the binary phase diagram ${ }^{[1]}$. Graph represent cloud points measured with conventional light scattering and showed good agreement with the optical image. The cloud points determined with this high-throughput method were validated by the good agreement with conventional light scattering results and single composition controls.

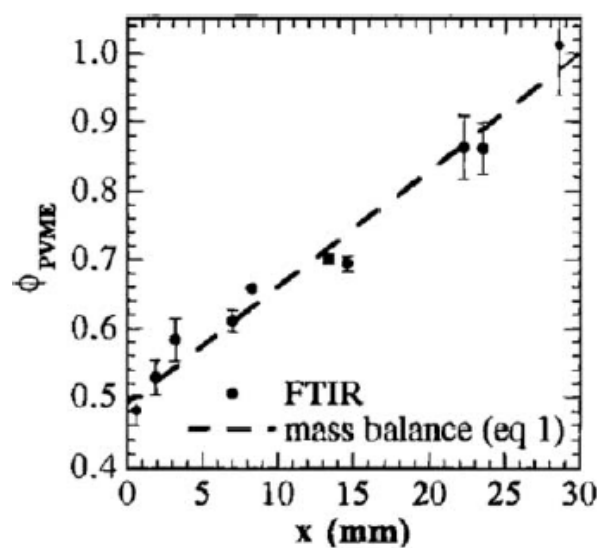

(a)

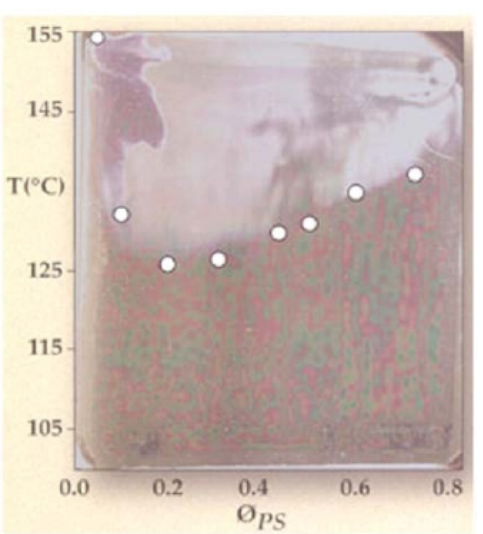

(b)

Fig. $2^{[1]}$ Determination of a polymer phase diagram from polymer film combinatorial spreads/libraries: (a) comparison of composition obtained from mass balance and direct measurement using FTIR showing good agreement for gradient films using the method shown in Fig.1(a)- (c) . (b) PS and PVME binary phase diagram showing the cloud points which separate the single-phase region from the two-phase region . 
Wang et al ${ }^{[2]}$ used the gradient hot-stage to effectively study the phase diagram of coexisting liquid-liquid phase separation and crystallization in nearly isorefractive blends of statistic ethylene/hexane (PEH) and ethylene/butene (PEB) copolymers. In addition to the composition gradient, a thickness gradient could be easily generated (Fig.1 (c)) to study its effect on polymer behavior, including thin film dewetting ${ }^{[3,4]}$, surface pattern formation ${ }^{[3,4,8]}$, and crystallization growth rate and morphology ${ }^{[9]}$. Above were the gradient film approach which was convenient for many binary polymer systems. However, several factors motivate the use of discrete combinatorial libraries for many polymeric systems ${ }^{[10]}$ : (i) expansion from binary to multicomponent systems; (ii) the study of behavior such as phase separation in the bulk, rather than in thin films which are known to be affected by surface interactions ${ }^{[11]}$; (iii) the investigation of low molecular mass mixtures or rubbery polymers at room temperature, whose large mobility would disrupt a composition gradient; (iv) the immobilization of composition libraries, which permits multiple, reversible, experimentation; and (v) the elimination of possible complications involving physical processes driven by composition (and thickness) gradients ${ }^{[10]}$.

Manish M Kulkarni and Alamgir Karim developed a combinatoral approach by use of Gradient composition mixer and thickness flow coater. Fig. 3 shows schematic of the fluid composition mixer and flow coated assembly. The polymer fluids to be mixed are pumped out from syringe pumps S1 and S2 to a passive chaotic mixer M. The mixed fluid paste/solution is drawn on the substrate and spread using the blade (B) to coat polymer film of desire thickness. Both the mixing and spreading rates can be programmed to obtain a composition as well as thickness gradient. The mixing and spreading process can be performed on-the-fly for low viscosity liquids. For higher viscosity (e.g. polymer pastes, elastomers) the mixed fluid tube is first drawn on the substrate and subsequently spread on the substrate using the flow coater.

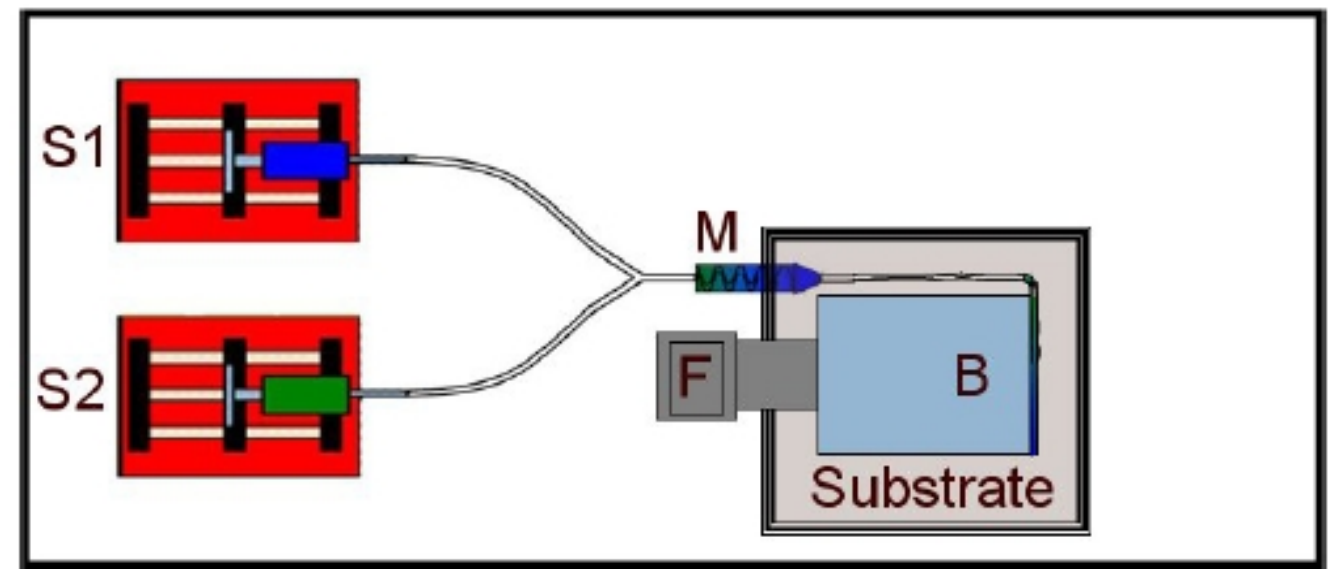

Fig. $3^{[12]}$ Schematic of combinatorial for polymer solution mixing and coating assembly

The whole process was like this: fluids to be mixed are pumped out from S1 and S2 syringe pumps. $\mathrm{M}$ was the passive chaotic mixer. $\mathrm{F}$ and $\mathrm{B}$ were stand and blade; components of a flow coater assembly. The mixed fluid paste/solution was drawn on the substrate and spread using the blade to coat polymer film of desire thickness. Both the mixing and spreading rates could be programmed to obtain a composition as well as thickness gradient.

The composition gradient film (CGF) or constant thickness film coated on a GSE substrate obtained as described earlier was placed on a thermal gradient stage (TGS) with one end connected to heating rod and the other end being cooled by flowing chilled fluid, such that the two gradients (e.g. CGF vs. temperature or GSE vs. temperature) were orthogonal to each other as shown in Fig. 2. $\mathrm{t} 1$ and $\mathrm{t} 2$ indicated total heating time. The shaded portions indicated a particular region of interest on the gradient film (e.g. phase transition, dewetting, adhesion etc.) 


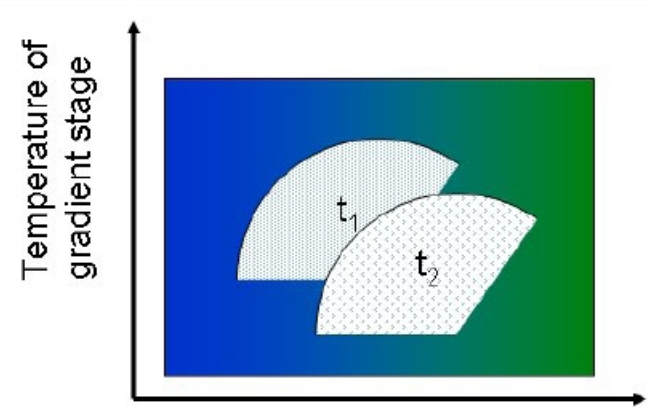

Polymer 1 : polymer 2 ratio

Fig. $4^{[12]}$ Schematic of CGF annealed orthogonal to a temperature gradient on a TGS. $\mathrm{t} 1$ and $\mathrm{t} 2$ indicated total heating time. The shaded portions indicate a particular region of interest on the gradient film (e.g. phase transition, dewetting, adhesion etc.)

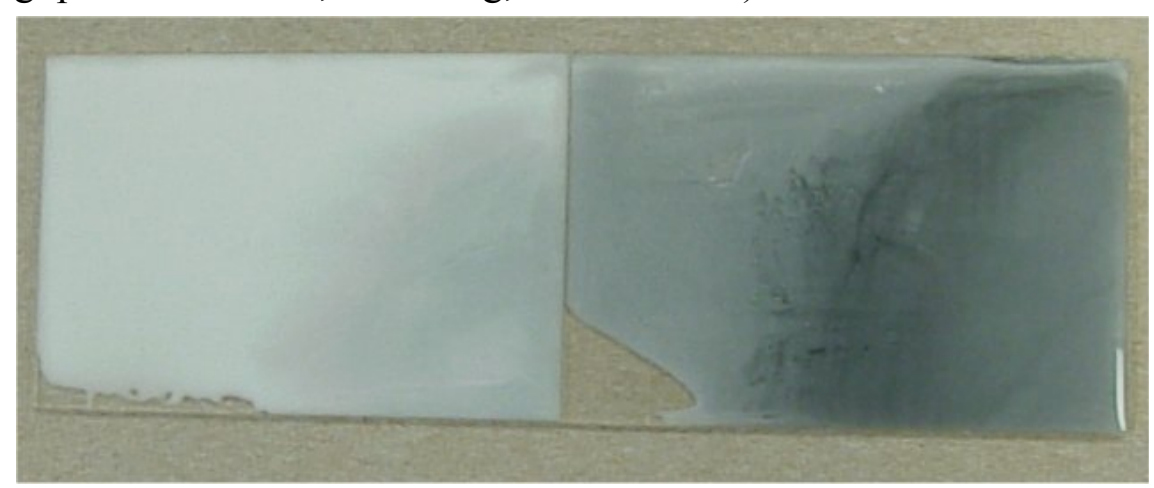

Fig.5 ${ }^{[12]}$ Composition gradient sample of PDMS elastomer (white) mixed with increasing percentage of crosslinker (black)

A typical composition gradient polymer films was shown in Fig. 7 Here a PDMS elastomer (white) was mixed with increasing percentage of crosslinker (black). This film was annealed on a TGS with the composition and temperature orthogonal to each other. The mechanical and adhesive properties of the film are currently being investigated for each of the unique composition and thermal point on the sample. Thus just one sample is sufficient to map out various properties of the polymer mix coating over entire composition and thermal range ${ }^{[13]}$.

Owing to the difficulty of separating the mixtures of different materials into discrete compositions, combinatorial approaches developed by the pharmaceutical industry dramatically changed the way leadcompounds for the development of new drugs are identified ${ }^{[14]}$. In the classical "CombiChem" approach, millions of moieties had been combinatorialy synthesized within a single reaction vessel before potentially active compounds were identified in a selective bioassay. This classical CombiChem approach for the discovery of materials couldn't be used for the discovery of lead polymers, owing to several conceptual and experimental difficulties discussed in this review. Instead, material scientists had produced a number of advances in synthesis and characterization that were leading towards a truly combinatorial synthetic and rapid screening characterization approach for the identification of structure-property correlations in polymethacrylates. The concept of using spatially resolved libraries was later adapted at the National Institute for Standards and Technology (NIST) where researchers explored the properties of polymer blends within a two-dimensional grid in which all possible blend compositionswere represented by a pair of XY coordinates ${ }^{[15-17]}$. Combinatorial approach used with computational modeling are together utilised in new-pharamacy materials discovery.

Concluded from above literatures, it was easily to be find that combinatorial approach began to 
be used in many chemical or industrial field so as to discover new kinds of polymers, especially in pharamarcy industry. For the materials science and engineering, combinatorial approach should base on the priciples of passive mixing and active mixing and pay more attention in studying the mixtures or where the phenonemen happened, dip out the micro- structures of mixtures and map the composition of mixtures.

\section{Conclusion and Research Prospection}

High-throughput and combinatorial methods have become increasingly popular in material discovery, characterization, and optimization. Combinatorial methods have several advantages over traditional techniques, including fast data acquisition, more thorough examination of experimental variables, equal processing conditions for a given specimen, and low experimental error ${ }^{[13,18]}$.

And the research prospection is as follows. First, this method is developped based on two kinds of reactive mechanisms, active mixing and passive mixing. However, it was the key point to control quantities of the discrete compositions and speed of blending the two kinds of compositions. Second, combinatorial approach is one kind of method of studying the micro-structures or properties of mixtures with two or more discrete compositions. The key was to research the exact properties of compositions according to standards and find the deeper relations between structures and properties.Finally, this method is limited to be used in discovering new kinds of non-metallic materials by small quantities, not suitable to large amounts of materials-production.

\section{References}

[1] Meredith JC, Karim A, Amis EJ. Macromolecules 2000;33:5760.

[2] Wang H, Shimizu K, Hobbie EK, Wang Z-G, Meredith JC, Karim A, et al. Macromolecules 2002;35:1072.

[3] Meredith JC, Karim A, Amis EJ. MRS Bull 2002;27:330.

[4] Meredith JC, Smith AP, Karim A, Amis EJ. Macromolecules 2000;33:9747.

[5] Bhagat AAS, Peterson ETK, Papautsky I. Journal of micromechanics and microengineering 2007; 17:1017-1024.

[6] Wang S, Huang X, Yang C. Lab on a chip 2011;11:2081-7.

[7] Meredith JC, Amis EJ, Karim A, Amis EJ. Macromolecules 2000;33:5760-5762.

[8] Smith AP, Douglas JF, Meredith JC, Amis EJ, Karim A. Phys Rev Lett 2001;87:015503.

[9] Beers KL, Douglas JF, Amis EJ, Karim A. Langmuir 2003;19:3935.

[10] Cabral JT, Karim A. Meas Sci Technol 2005;16:191.

[11] Sung L, Karim A, Douglas JF, Han CC. Phys Rev Lett 1996;76:436.

[12] Manish M Kulkarni, Alamgir Karim. Combinatorial Techniques for Viscous Polymer Solution Mixting and Coating. Department of Polymer Engineering, The University of Akron.

[13] Paul F. Holmes, Mike Bohrer, Joachim Kohn. Exploration of Polymethacrylate Structure-property Correlations: Advances Towards Combinatorial and High-throughput Methods for Biomaterials Discovery. Progress in Polymer Science, 2008(33): 787-796.

[14] Lowe G. Combinatorial chemistry. Chem Soc Rev 1995;24:309-17.

[15] Smith AP, Douglas JF, Meredith JC, Amis EJ, Karim A. Combinatorial study of surface pattern formation in thin block copolymer films. Phys Rev Lett 2001;87, 015503(1-4).

[16] Smith AP, Douglas JF,Meredith JC, Amis EJ, Karim A. High-throughput characterization of pattern formation in symmetric diblock copolymer films. J Polym Sci, Part B: Polym Phys 2001;39:2141-58. 
[17] Meredith JC, Smith AP, Karim A, Amis EJ. Combinatorial materials science for polymer thin-film dewetting. Macromolecules 2000;33:9747-56.

[18] Amis EJ. Combinatorial materials science - reaching beyond discovery.

Nat Mater 2004;3:83-5. 